\title{
Paraplegia after Lung Cancer Resection
}

Fayçal El Oueriachi ${ }^{1}$, Mustapha Bensghir ${ }^{2}$, Omar Slaoui ${ }^{1}$, Elassane Ayouba ${ }^{1}$, Mehdi Samali ${ }^{2}$, Mohamed Massine El Hammoumi ${ }^{1}$, El Hassane Kabiri ${ }^{1}$

Departments of ${ }^{1}$ Thoracic Surgery and ${ }^{2}$ Anesthesiology; Mohamed V Military Teaching Hospital, Faculty of Medicine and Pharmacy, Mohamed V University, Rabat, Morocco.

\section{Corresponding Author:}

Dr Fayçal El Oueriachi

Email: faycaldr@hotmail.com

This is an Open Access article distributed under the terms of the Creative Commons Attribution License (creativecommons.org/ licenses/by/3.0).

Received

Accepted

Published

January 7,2020

July 1,2020

July 15,2020

\begin{abstract}
Background: The major causes of post-operative morbidity and mortality following lung resection are cardiovascular and pulmonary complications. Neurological complications as phrenic or recurrent nerve injury are rarely found, paraplegia is exceptional. Case Report: We report a case of 61 year old man who presented a paraplegia discovered incidentally in the immediate aftermath of a lobectomy for lung cancer. CT showed a possible intra-cord migration of an epidural catheter. The catheter was immediately removed and neurological recovery was progressive and complete. Conclusion: We want to identify the causes, anatomical and technical reasons leading to this complication, recall the necessary measures to prevent its occurrence and finally stress the importance of seeking signs of neurological impairment during the puncture and catheter placement, also after surgery when the effects of general anesthesia are abolished.
\end{abstract}

Keywords: Epidural Anesthesia, Lung Neoplasms, Paraplegia, Punctures, Thoracic Surgery.

\section{Introduction}

Thoracic epidural analgesia is routinely used for its several benefits in opened thoracic surgery. In addition to its superlative post-operative analgesic effect, it definitely improves respiratory and hemodynamic functions. Occurrence of spinal cord or nerve root injury during epidural catheterization is often accompanied by lightening paresthesia or lancinating pain at the moment of puncture or catheter placement [1].

We report the case of a patient who developed paraplegia due to a possible spinal cord misplacement of a catheter following an apparent successful thoracic epidural analgesia. The paraplegia was diagnosed fortuitously in the aftermath of lung cancer surgery. Recovery was observed shortly after removal of the catheter. Through this case we recall the devastating neurological consequences we can avoid by following technical precautions and ensuring that patients are responsive after disappearance of general anesthesia and neuromuscular block effects.

\section{Case Report}

A 61 year-old man (weight $=71 \mathrm{~kg}$, height $=179$ $\mathrm{cm}$ ) was referred to the thoracic surgery department for surgery of pre-operative stage IIb right upper lobe lung cancer. He had no pathological history. The pre-anesthetic consultation showed that the examination of the thoracic spine was unremarkable, similarly no neurological abnormalities were noted. The rest of physical and para-clinical investigations were normal. After a pre-operative fasting and pre-medication with hydroxyzine (75 mg) the patient was admitted in the operating room. A standard monitoring with heart rate (HR), non-invasive blood pressure (BP) and blood oxygen saturation $\left(\mathrm{SPO}_{2}\right)$ was installed. Peripheral 
venous access was secured by $16 \mathrm{G}$ catheter and infusion of saline solution $(0.09 \%)$ was started. The initial parameters $\left(\mathrm{HR}, \mathrm{BP}\right.$ and $\mathrm{SPO}_{2}$ ) were within normal limits. The establishment of an epidural analgesia system was decided before starting general anesthesia; for this, the patient was kept in sitting position and the procedure was explained to him step by step. After identification of the interspinous space T6 and preparation of the skin with an iodine solution (Betadine), a local anesthesia was made by infiltration of $5 \mathrm{ml}$ of lidocaine $2 \%$ via $21 \mathrm{G}$ needle. With the midline technique, a $17 \mathrm{G}$ Tuohy needle was introduced with air for the lossof-resistance test. Five attempts were necessary for the identification of the epidural space. Two of these attempts were made by a para-midline technique. An epidural catheter was inserted 4 $\mathrm{cm}$ easily. No paresthesia was observed during needle or catheter insertion. A test dose of $3 \mathrm{ml}$ of local anesthetic (lidocaine $2 \%$ with $1 / 2,00,000$ epinephrine) was injected via epidural catheter to exclude intra-vascular or sub-arachnoid injection. After a negative test dose (no changes in the HR or BP and no motor blockage), infusion of a solution containing $0.125 \%$ of bupivacaine and fentanyl $3 \mu \mathrm{g} / \mathrm{mL}$ via the epidural catheter was started at a rate of $4 \mathrm{ml} / \mathrm{h}$.

Upper right lobectomy with right mediastinal lymphadenectomy was performed through the fifth intercostal space. The operative time was 3 hours during which blood loss was approximately $300 \mathrm{ml}$. The patients $\mathrm{HR}, \mathrm{BP}$ and $\mathrm{SPO}_{2}$ remained stable. At the end of the surgery, the patient was awakened and extubated in the operative room. He was observed after awakening in the post-operative room for 2 hours; he expressed no complaint, was pain-free and his vital parameters were normal. The patient was then returned to the thoracic surgery department where a routine post-operative clinical examination showed that the lower limbs were completely paralyzed and non responsive to any stimulation. A neurological evaluation revealed complete sensory and motor loss below
T8 dermatome level, motor and plantar reflexes were abolished in both lower limbs. In presence of these symptoms, we first suspected a spinal cord compression with an epidural hematoma. We interrupted the administration of bupivacaine through the epidural catheter and spinal CT scan, performed 3 hours after surgery, showed that the catheter had an ascendant trajectory in the sub-arachnoid space; it crossed the spinal cord from right to left on a length of $3 \mathrm{~cm}$ [Fig.1]. An intra-cord catheterization with a subsequent local anesthetic injection was highly suspected. The catheter was immediately removed to minimize the damage of possible spinal cord trauma. A bolus of $200 \mathrm{mg}$ /day of corticosteroids was administered thereafter for 3 days to treat or prevent a possible bone marrow edema. Neurological recovery was observed few hours later and was assessed by a consultant neurologist, it was progressive, initially sensitive and motor. One week after removal of the catheter, neurological recovery was complete and the ability to walk had recovered fully.

MRI, performed 48 hours after surgery, showed several small collections of air in the epidural space [Fig.2], the thoracic cord appeared
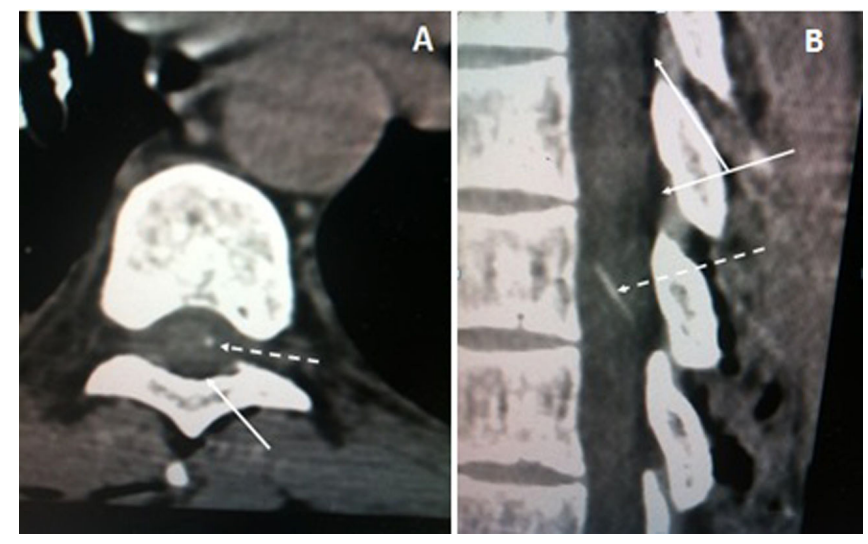

Fig.1 (a): Axial view of spinal CT scan showing the catheter (discontinuous arrow) contacting the cord at the D7-D8 level and an air collection in the posterior part of the epidural space (continuous arrow); (b): Sagittal view showing the spinal cord, possibly, entered by the catheter on $3 \mathrm{~cm}$ of length. Bubbles of air are clearly visible in subcutaneous and epidural spaces. 
with no abnormalities. One month later, clinical examination had shown no neurological deficits.

\section{Discussion}

Thoracic epidural analgesia (TEA) remains the best choice to ensure a rapid and effective control of pain which also aims to reduce post-operative respiratory mechanical dysfunction impact after thoracic surgery. A good quality of analgesia is then obtained by a continuous administration of a local anesthetic through the epidural catheter. The overall incidence of complications following TEA has been reported as 3\% [2]. Technique-related complications of TEA including spinal cord injury, breaking or subarachnoid migration of an epidural catheter, pneumothorax and hemothorax are very rare [3]. Spinal cord misplacement of an epidural catheter is unusual, as far as we know there is only one reported case of an epidural catheter that had possibly entered the cord [4].

Paraplegia following thoracotomy as a complication of surgery is estimated to be approximately $0.1 \%$ [5], its main causes are extensive dissection in the posterior angle of postero-lateral thoracotomy, traumatic excision of the posterior extremity of the ribs and thrombosis of the anterior spinal artery after ligation of intercostal vessels. Although rare, this etiology should be considered in the differential diagnosis in such cases. The majority of reported cases of spinal cord trauma after epidural catheterization were observed in patients who underwent epidural analgesia under general anesthesia [6]. In conscious patients this technique is usually accompanied by paresthesia or pain during puncture [7], these symptoms are a warning that needle or catheter had touched a nervous structure following the attempt to identify epidural space by loss-of-resistance test. The lack of these symptoms in our patient is possibly related to the spread of local anesthetic into the epidural and sub-arachnoid space before inserting the catheter. A secondary migration of the catheter within the spinal cord after general anesthesia induction,

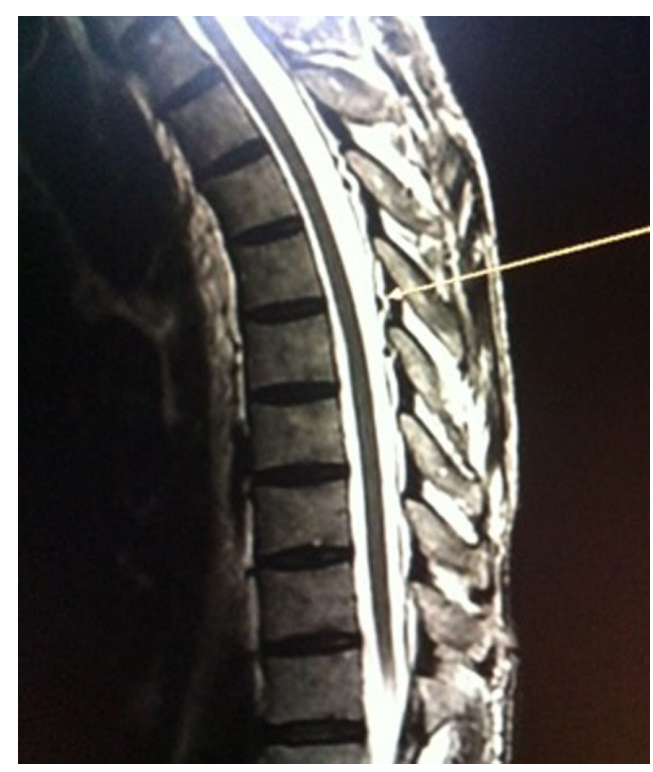

Fig.2: MRI sagittal view of the thoracic spinal column 48 hours after surgery displaying air bubbles in the epidural space. The spinal cord shows normal signals.

favored by anatomical discontinuity of the thoracic ligamentum flavum [8] and a dural breach caused by the needle is a theory which could explain the lack of response of the patient during insertion of the catheter.

The negativity of aspiration test of blood and cerebrospinal fluid is in favor of the second theory. Although it is exceptional, we report here another case where traumatic epidural catheter insertion had caused no warning signs that would indicate the interruption of the technique. Other precautions in the practice of EA to reduce the risk of spinal cord injury were mentioned in some publications; shorter needles are preferable to infiltrate local anesthetic agents with the objective of not exceeding $4 \mathrm{~cm}$ during the penetration by the needle because in thin patients the distance between the skin and the epidural space could be inferior to $3 \mathrm{~cm}$ [9]. The procedure should be interrupted after several unsuccessful attempts [10], thus, in such patients a safe needle insertion can be guided by ultrasonography. The use of ultrasound allows to appreciate the depth of epidural space, and to reduce the number of attempts and time of 
completion of the technique. The same, incidence of inadvertent dural puncture, vascular puncture and paresthesia was low with ultrasound. With this technique the success rate is higher with a lower post-block pain scores and better patient satisfaction $[11,12]$. Patients with abnormal spinal anatomy, difficulty of realization of epidural anesthesia are often noted. In these cases, the use of ultrasound in first intention is justified. In our patient, the examination of the spine was normal, and there were no unforeseen difficulties that could indicate the use of ultrasonography in second line.

The technique of loss-of-resistance in the detection of epidural space using air in the syringe is being abandoned in favor of saline [13]. The presence of an air volume more than $2 \mathrm{ml}$ in the spinal canal is provider of major complications, such as cord compression and pneumocephalus. In our patient the anesthetist used air, gas bubbles were contained in the epidural space and there were no related neurological complications after removal of the catheter. Serious but rare complications including permanent paraplegia, seizures and cardiac arrest can occur [7]. In our case, paraplegia was transient. The progressive and complete neurological recovery could be related to the absence of major tissue damage and ischemic effects in the spinal cord, as confirmed by MRI findings. Therefore, paraplegia would be more related to intra-spinal administration of local anesthetics than the intra-cord catheterization and injury.

\section{Conclusion}

Complications related EA in thoracic surgery can be devastating and must be known by all practitioners. This case illustrates the importance of taking all precautions in the practice of this technique, the possibility of lack of warning signs in conscious patients after traumatic EA, and the necessity of post-operative neurological evaluation for early diagnosis of complications and institution at time of salvage procedures [15].
Contributors: El Oueriachi Fayçal, Bensghir Mustapha and Kabiri El Hassane contributed to the redaction of the manuscript; Slaoui Omar, Elassane Ayouba, Samali Mehdi, El Hammoumi Mohamed Massine: prepared the figures and the literature research. El Oueriachi Fayçal will act as a study guarantor. All authors approved the final version of the manuscript and are responsible for all aspects of the study.

Funding: None; Competing interests: None stated.

\section{References}

1. Bomberg H, Paquet N, Huth A, Wagenpfeil S, Kessler P, Wulf $\mathrm{H}$, et al. Epidural needle insertion: A large registry analysis. Anaesthesist. 2018;67:922-930.

2. Giebler RM, Scherer RU, Peters J. Incidence of neurologic complications related to thoracic epidural catheterization. Anesthesiology. 1997;86:55-63.

3. Chadwick VL, Jones M, Poulton B, Fleming BG. Epidural catheter migration: a comparison of tunnelling against a new technique of catheter fixation. Anaesth Intensive Care. 2003;31:518-522.

4. Katz N, Hurley R. Epidural anesthesia complicated by fluid collection within the spinal cord. Anesth Analg. 1993;77:1064-1065.

5. Attar S, Hankins JR, Turney SZ, Krasna MJ, McLaughlin JS. Paraplegia after thoracotomy: report of five cases and review of the literature. Ann Thorac Surg. 1995;59:14101416.

6. Bromage PR, Benumof JL. Paraplegia following intracord injection during attempted epidural anesthesia under general anesthesia. Reg Anesth Pain Med. 1998;23:104-107.

7. Auroy Y, Narchi P, Messiah A, Litt L, Rouvier B, Samii $\mathrm{K}$. Serious complications related to regional anesthesia: results of a prospective survey in France. Anesthesiology. 1997:87:479-486.

8. Lirk P, Kolbitsch C, Putz G, Colvin J, Colvin HP, Lorenz I, et al. Cervical and high thoracic ligamentum flavum frequently fails to fuse in the midline. Anesthesiology. 2003;99:1387-1390.

9. Greiff J, Cousin MJ. Subarachnoid and extradural anesthesia. In: Nimmo WS, Rowbotham DJ, Smith G, editors. Anesthesia, $2^{\text {nd }}$ ed. Oxford: Blackwell Scientific. 1994; pp.1411-54.

10. Kleinman W, Mikhail M. Spinal, epidural, and caudal Blocks. In: Morgan GE, Mikahil M, Murray MJ, editors. Clinical Anaesthesiology. $4^{\text {th }}$ ed. NewYork: Tata McGraw-Hill; 2009. pp. 320.

11. Rasoulian A, Lohser J, Najafi M, Rafii-Tari H, Tran D, Kamani AA, et al. Utility of prepuncture ultrasound for localization of the thoracic epidural space. Can J Anesth. 2011;58:815-823.

12. Chin KJ1, Karmakar MK, Peng P. Ultrasonography of the adult thoracic and lumbar spine for central neuraxial blockade. Anesthesiology. 2011;114:1459-1485. 
13. Shenouda PE, Cunningham BJ. Assessing the superiority of saline versus air for use in the epidural loss of resistance technique: a literature review. Reg Anesth Pain Med. 2003;28:48-53.

14. Ferrante E, Rubino F, Porrinis L. Pneumocephalus: A rare complication of epidural catheter placement during epidural blood patch. Headache. 2014;54:539-540.

15. Bracken MB, Holford TR. Effects of timing of methylprednisolone or naloxone administration on recovery of segmental and long tract neurological function in NASCIS 2. J Neurosurg. 1993;79:500-507. 